\title{
Enfances de classe : de l'inégalité parmi les enfants
}

Bernard Lahire (dir), Seuil, 2019, 1232 p.

Jean-Pierre Véran

\section{OpenEdition}

Journals

Édition électronique

URL : https://journals.openedition.org/ries/8957

DOI : $10.4000 /$ ries.8957

ISSN : 2261-4265

Éditeur

France Education international

Édition imprimée

Date de publication : 1 décembre 2019

ISBN : 9782854206258

ISSN : $1254-4590$

Référence électronique

Jean-Pierre Véran, "Enfances de classe : de l'inégalité parmi les enfants », Revue internationale d'éducation de Sèvres [En ligne], 82 I décembre 2019, mis en ligne le 01 décembre 2019, consulté le 13 janvier 2022. URL : http://journals.openedition.org/ries/8957 ; DOI : https://doi.org/10.4000/ries.8957

Ce document a été généré automatiquement le 13 janvier 2022.

(c) Tous droits réservés 


\section{Enfances de classe: de l'inégalité parmi les enfants}

Bernard Lahire (dir), Seuil, 2019, 1232 p.

Jean-Pierre Véran

\section{RÉFÉRENCE}

Enfances de classe : de l'inégalité parmi les enfants, Bernard Lahire (dir), Seuil, 2019, 1232 p.

1 S'il fallait trouver un autre titre à cet ouvrage, on pourrait l'intituler Premières socialisations enfantines, étude de cas française. En effet, la question qui y est traitée concerne tous les enfants et tous les systèmes éducatifs du monde. Partout sur la planète, les conditions matérielles de logement, d'alimentation, de santé, d'accès aux services, mais aussi la langue parlée dans la famille, les loisirs pratiqués, les objets culturels familiers, les relations sociales constituent de forts déterminants de la socialisation enfantine préscolaire et préélémentaire.

Centré sur dix-huit études de cas d'enfants scolarisés en grande section de maternelle en France, cet ouvrage témoigne de la diversité de la population scolaire, en termes de classes sociales, certes, mais aussi d'origine. Parmi les quatre enfants issus des classes populaires dont l'étude de cas est présentée dans l'ouvrage, Libertad est fille de parents rom venus de Roumanie et parlant hongrois, Asham a pour mère une infirmière formée au Sri Lanka, Ilyes un père né en Algérie, comme ses grands-parents maternels. En cela, cet ouvrage collectif parle d'élèves de l'école française, qui sont aussi, dans les classes populaires notamment, des enfants originaires de divers pays du monde.

3 Par-delà ces origines internationales, les lecteurs de la revue trouveront aussi un intérêt au caractère sans précédent de cette étude. Ce travail scientifique, mené par un collectif de dix-sept chercheuses et chercheurs entre 2014 et 2018, s'est voulu à hauteur d'enfant, pour nous donner à voir et à ressentir, tout autant qu'à comprendre, la diversité des conditions dans lesquelles vivent les 35 enfants faisant l'objet d'une étude de cas, parmi lesquels dix-huit ont été publiées dans le livre. 
4 Ici, on ne se contente pas de noter la catégorie socio-professionnelle des parents ou l'indice de position sociale des enfants qui ont été choisis pour avoir dans l'échantillon une représentation optimale des classes et fractions de classes sociales. Par de multiples entretiens, les auteurs ont rencontré une pluralité d'acteurs (parents, famille, adulte significatif, camarades, enseignants, nourrice), permettant de percevoir précisément le capital économique et financier de la famille, les ressources scolaires et culturelles mais aussi résidentielles, corporelles, sanitaires, langagières, esthétiques, mobilisées autour de chaque enfant. Ils ont, au travers de ces échanges, observé de multiples domaines et pratiques. Ils ont accompagné en observation ethnographique les enfants pendant une journée entière à l'école et leur ont proposé des jeux langagiers et narratifs.

5 Si les uns accumulent des ressources matérielles, morales, culturelles, langagières, scolaires, corporelles, sanitaires, les autres cumulent des handicaps et des obstacles dans ces mêmes domaines. Les enfants ne choisissent ni le terrain sur lequel ils jouent ni la nature du jeu...

$6 \quad$ La finesse de l'analyse ne se résout pas à la partition entre enfants appartenant aux classes populaires, aux classes moyennes ou aux classes favorisées. Nul fixisme, nul simplisme dans la classification, que l'hétérogamie rend parfois délicate; de multiples nuances permettent de saisir comment, au sein même d'une classe, on se distingue de ceux qui sont les plus proches pour tendre vers ceux qui sont de l'autre côté de la frontière sociale et comment on peut vivre en tension permanente une position à cheval entre deux classes.

7 On ne retiendra ici que certaines lignes de force parmi d'autres. Au chapitre de l'enfance des inégalités, comment ne pas insister sur les écarts sanitaires entre des enfants confrontés aux conduites alimentaires de la pauvreté quand d'autres apprennent les pratiques de santé comme un style de vie? Comment ne pas évoquer les écarts vestimentaires, les fossés séparant les loisirs populaires des loisirs bourgeois, la place différente du langage dans les relations familiales, les enfants maîtrisant l'autocontrôle quand d'autres ne connaissent que la punition de leurs bêtises, l'impact des expériences ou inexpériences scolaires des parents, l'instabilité résidentielle des uns rythmée par les expulsions, quand d'autres partagent leur temps entre résidences principale, secondaire et vacances à l'étranger, les attentes différenciées des enseignants à l'égard des enfants en fonction de la précarité ou de la solidité du milieu familial? Tous ces enfants fréquentent des écoles maternelles, ils sont tous en grande section, pourtant l'école publique d'un arrondissement privilégié de Paris ne ressemble pas à celle d'un quartier populaire d'une ville de province ou d'un bourg rural, et ils arrivent tous à l'école en vivant dans des mondes différents.

8 La question qui est au cœur de cette recherche, c'est l'accès socialement différencié à toutes les extensions de soi possibles pour chaque enfant. Bernard Lahire écrit ainsi dans sa conclusion :

Disposer de plus d'espace, de plus de temps, de plus de confort matériel, de plus d'aide humaine, de plus de connaissances, de plus d'expériences esthétiques, de plus d'informations, de plus de soins, de plus de vocabulaire et de formes langagières, de plus de possibilités de se vêtir, de se reposer ou de se divertir, et bien-sûr, avoir plus d'argent (...) pour pouvoir accéder à toutes les formes de ressources, des biens matériels aux biens culturels, en passant par les divers services domestiques, éducatifs, médicaux, techniques, etc., c'est avoir plus de pouvoir sur le monde et sur autrui. 
L'auteur en vient à penser que des enfants biologiquement égaux qui naissent dans des milieux familiaux extraordinairement différents, «ne sont pas vraiment les mêmes enfants ». Dans une même société, certains vivent une réalité augmentée, d'autres une réalité diminuée.

10 En cela, cette étude sociologique d'envergure fait œuvre politique. Est-il admissible que l'enrichissement des uns se fonde sur l'appauvrissement des autres? Bernard Lahire suggère que, s'il est difficile de parvenir à un partage équitable du capital économique et financier, il est sans doute plus à la portée des États d'assurer une transmission à tous du patrimoine culturel et de garantir à chacun de bonnes conditions sanitaires.

11 On ne peut s'empêcher, au fil de la lecture, de se répéter l'article premier de la Déclaration des droits de l'homme et du citoyen votée en 1789 par l'Assemblée nationale française, «Les hommes naissent et demeurent libres et égaux en droits. Les distinctions sociales ne peuvent être fondées que sur l'utilité commune ", et à l'article premier de la Déclaration universelle des droits de l'homme votée par l'Organisation des Nations Unies en 1948 :

Tous les êtres humains naissent libres et égaux en dignité et en droits. Ils sont doués de raison et de conscience et doivent agir les uns envers les autres dans un esprit de fraternité.

12 On ne peut non plus s'empêcher de penser que cette étude, portant sur des élèves de 5-6 ans en France, donne à voir des écarts qui ne sont pas propres à la société française, mais à bien des sociétés sur tous les continents. On est alors plus conscient encore de ce qui se joue à l'école, dès les premières années puis tout au long de l'enfance et de l'adolescence, jusqu'à l'âge adulte. Si certains élèves sont plus à l'aise que d'autres, parce qu'ils ont confiance en eux, si certains mêmes peuvent faire preuve d'arrogance quand d'autres se tiennent en retrait ou manifestent leur rejet des activités scolaires, c'est sans doute aussi parce que les uns ont acquis dès leur naissance le sentiment de leur légitimité et des compétences de leadership, quand d'autres éprouvent le sentiment que l'école n'est pas faite pour eux, parce que ce qui s'y enseigne et s'y apprend est étranger à leur univers.

On pourrait penser qu'il n'y a là rien de neuf, quand, pour s'en tenir à la sociologie française de l'école, dans Les Héritiers (1964) et La Reproduction (1970) Bourdieu et Passeron posaient les éléments d'une théorie du système d'enseignement, et quand Baudelot et Establet décrivaient L'école capitaliste en France (1971) et affirmaient que L'école primaire divise (1975) ou déconstruisaient L'Elitisme républicain (2009). On pourrait ajouter qu'en 2015, le rapport remis à la ministre de l'éducation nationale française intitulé Grande pauvreté et réussite scolaire, le choix de la solidarité pour la réussite de tous ${ }^{1}$, attirait l'attention sur les fragilités multiples des enfants pauvres, l'inégalité entre enfants pour répondre aux exigences scolaires, et préconisait d'ouvrir des chemins pour donner à tous les élèves une culture commune. L'OCDE elle-même ne serait pas en reste, puisqu'en 2018, le rapport Equity in Education Breaking down barriers to social mobility ${ }^{2}$ a montré qu'en moyenne, dans les pays de l'OCDE pour lesquels on dispose de données comparables, plus des deux tiers de l'écart de résultats scolaires observé à l'âge de 15 ans et environ deux tiers de l'écart constaté chez les 25-29 ans avaient déjà été relevés chez les enfants de 10 ans.

Pourtant, il n'en est rien. D'une part, parce que l'étude se situe en amont de l'école élémentaire. D'autre part, parce que le travail du collectif de recherche coordonné par Bernard Lahire va plus loin: il éclaire les mécanismes profonds de production des 
inégalités dès la naissance au sein d'une société, et les écarts sidérants qui se creusent au fil du temps entre les vies des enfants d'une même société. Il le fait en faisant partager aux lecteurs les réalités concrètes et symboliques de vies d'enfants si différentes.

15 Quelle que soit sa nationalité ou le pays où il exerce, le lecteur de cet ouvrage collectif de 1200 pages sera outillé pour mieux appréhender les premières socialisations enfantines et l'enjeu démocratique crucial que constitue l'inégalité parmi les enfants.

\section{NOTES}

1. Jean-Paul Delahaye (2015). Grande pauvreté et réussite scolaire, le choix de la solidarité pour la réussite de tous. Inspection générale de l'éducation nationale, mai.

2. [http://www.oecd.org/education/equity-in-education-9789264073234-en.htm].

\section{AUTEUR}

\section{JEAN-PIERRE VÉRAN}

Jean-Pierre Véran est inspecteur d'académie - inspecteur pédagogique régional $(\mathrm{H})$, membre du comité de rédaction de la Revue internationale d'éducation de Sèvres et expert auprès de France Éducation international. Il intervient sur les questions de gouvernance des organisations éducatives, de politiques éducatives et d'éducation aux médias et à l'information. Auteur de plusieurs ouvrages, il tient également un blog consacré à l'éducation sur Mediapart : http:// blogs.mediapart.fr/blog/jean-pierre-veran/ ; courriel : jeanpierreveran2@gmail.com 